\title{
DIRECT SIMULATIONS OF HELICAL HALL-MHD TURBULENCE AND DYNAMO ACTION
}

\author{
Pablo D. Mininni ${ }^{1}$ \\ Advanced Study Program, National Center for Atmospheric Research, P.O.Box 3000, Boulder CO 80307, USA. \\ and \\ Daniel O. Gómez ${ }^{2}$ \\ Departamento de Física, Facultad de Ciencias Exactas y Naturales, Universidad de Buenos Aires, \\ Ciudad Universitaria, 1428 Buenos Aires, Argentina. \\ dgomez@df.uba.ar \\ and \\ Swadesh M. Mahajan \\ Institute for Fusion Studies, The University of Texas, Austin, Texas 78712, USA.
}

\begin{abstract}
Direct numerical simulations of turbulent Hall dynamos are presented. The evolution of an initially weak and small scale magnetic field in a system maintained in a stationary turbulent regime by a stirring force at a macroscopic scale is studied to explore the conditions for exponential growth of the magnetic energy. Scaling of the dynamo efficiency with the Reynolds numbers is studied, and the resulting total energy spectra are found to be compatible with a Kolmogorov type law. A faster growth of large scale magnetic fields is observed at intermediate intensities of the Hall effect.
\end{abstract}

Subject headings: MHD — magnetic fields — stars: magnetic fields — stars: neutron — accretion disks

\section{INTRODUCTION}

In recent years, the relevance of two-fluid effects has been pointed out in several astrophysical (Balbus \& Terquem 2001; Sano \& Stone 2002; Mininni, Gómez, \& Mahajan 2002, 2003a) as well as laboratory plasmas (Mirnov, Hegna, \& Prager 2003). The standard magnetohydrodynamic (MHD) framework for the study of astrophysical plasmas may not be adequate in the presence of strong magnetic fields and/or low ionization; the electric conductivity then is not isotropic and nonlinear effects arise in Ohm's law. In

\footnotetext{
${ }^{1}$ also at Departamento de Física, Facultad de Ciencias Exactas y Naturales, Universidad de Buenos Aires, Ciudad Universitaria, 1428 Buenos Aires, Argentina.

2 also at Instituto de Astronomía y Física del Espacio, Ciudad Universitaria, 1428 Buenos Aires, Argentina.
}

low temperature accretion disks around young stellar objects or in dwarf nova systems in quiescence, for example, the plasma is only partially ionized with a small abundance of charged particles. As a result, two new effects appear in Ohms's law: ambipolar diffusion and Hall currents. The predominance of either of these effects is determined by the ionization fraction and the plasma density (Sano \& Stone 2002; Braginskii 1965). The impact of ambipolar diffusion on dynamo action has been already studied by Zweibel (1988) (see also Brandenburg \& Subramanian (2000)). The Hall effect affects the dynamics of protostellar disks (Balbus \& Terquem 2001). In neutron stars magnetic fields are so strong that the Hall term can be even more important than the induction term for the magnetic field evolution (Muslimov 1994). The Hall effect is also known to be relevant in others astrophysical scenarios (Sano 
\& Stone 2002; Mininni, Gómez, \& Mahajan 2003b, and references therein).

The Hall effect, when strong, is expected to seriously affect the MHD results on the generation of magnetic fields in astrophysical and laboratory plasmas by inductive motions in a conducting fluid (dynamo effect). More specifically, it is expected to modify the growth and evolution of magnetic energy, since the addition of the Hall term to the MHD equations leads to the freezing of the magnetic field to the electron flow (in the non-dissipative limit) rather than to the bulk velocity field. The first studies on the impact of Hall currents on dynamo action (Helmis 1968; Helmis 1971) were carried out using mean field theory and the first-order smoothing approximation (Krause \& Rädler 1980). Helmis obtained decreasing dynamo action as the strength of the Hall terms increased. Recently, the impact of the Hall effect has been studied using the kinematic approximation or focusing on particular geometries, both in numerical simulations (Galanti, Kleeorin, \& Rogachevskii 1994) and in experimental and theoretical studies (Heintzmann 1983; Ji 1999; Rheinhardt \& Geppert 2002; Mirnov, Hegna, \& Prager 2003). A general closure scheme was also proposed to compute the contribution of the Hall term to the dynamo action $\alpha$-effect (Mininni, Gómez, \& Mahajan 2002) using mean field theory and the reduced smoothing approximation (Blackman \& Field 1999); it was found that the Hall effect could either suppress or enhance dynamo action. Expressions for the turbulent diffusivity using this closure were also derived for particular cases in Mininni, Gómez, \& Mahajan (2003a).

In the present work, we report results from direct numerical simulations of the dynamo action in MHD and Hall-MHD at moderate Reynolds numbers with strong kinetic helical forcing. The study of the scaling of the dynamo efficiency with increasing Reynolds numbers is the main aim of this paper. In a previous paper (Mininni, Gómez, \& Mahajan 2003b), we showed that three distinct dynamo regimes can be clearly identified: (1) dynamo activity is enhanced (Hall-enhanced regime), (2) it is inhibited (Hall-suppressed regime), (3) it asymptotically approaches the MHD value (MHD regime). These regimes arise as a result of the relative ordering between the relevant lengthscales of the problem. Namely, the energy-containing scale of the flow, the Hall length, and the correlation length of the magnetic seed. Simulations in Mininni, Gómez, \& Mahajan (2003b) were performed with $64^{3}$ spatial grid points and fixed Reynolds numbers. This study left unanswered the question whether the enhancement of dynamo action by Hall effect would increase or decrease with increasing Reynolds numbers and scale separation.

Theoretical estimates using mean field theory and a particular choice for the small scale fields suggest that the efficiency of Hall-MHD dynamos compared with the MHD counterpart will increase as the scale separation is increased (Mininni, Gómez, \& Mahajan 2002). In this work we present direct simulations with higher spatial resolutions and Reynold numbers that confirm this result. Also, the magnetic, kinetic, and total energy spectra developed in Hall-MHD turbulence are calculated and studied. These spectra correspond to the dynamo regime, where no imposed currents are present, i.e, the magnetic fields are purely self-generated. This regime is rather general and probably common to most astrophysical flows (Haugen, Brandenburg \& Dobler 2004).

The paper is organized as follows. In Section 2 we present the general equations describing the evolution of the fields. In Section 3, the code used to numerically integrate the Hall-MHD system is described, and all the simulations made with different resolutions and parameters are listed. Section 4 presents the results obtained in the MHD limit of our equations; the results obtained are similar to those of other authors (Meneguzzi, Frisch, \& Pouquet 1981; Brandenburg 2001), and provide a reference set to compare the Hall-MHD solutions with. Section 5 is devoted to the results of Hall-MHD simulations. In Section 6 we discuss a subset of MHD and Hall-MHD simulations made to study the evolution of large scale magnetic fields. Finally, in Section 7, we give a brief summary of the current effort.

\section{THE HALL-MHD SYSTEM}

Incompressible Hall-MHD is described by the modified induction and the dissipative Navier-Stokes equation,

$$
\begin{aligned}
\frac{\partial \boldsymbol{B}}{\partial t}= & \nabla \times[(\boldsymbol{U}-\epsilon \nabla \times \boldsymbol{B}) \times \boldsymbol{B}]+\eta \nabla^{2} \boldsymbol{B}(1) \\
\frac{\partial \boldsymbol{U}}{\partial t}= & -(\boldsymbol{U} \cdot \nabla) \boldsymbol{U}+(\boldsymbol{B} \cdot \nabla) \boldsymbol{B}- \\
& -\nabla\left(P+\frac{B^{2}}{2}\right)+\boldsymbol{F}+\nu \nabla^{2} \boldsymbol{U} \\
\nabla \cdot \boldsymbol{B}= & 0=\nabla \cdot \boldsymbol{U},
\end{aligned}
$$


where $\boldsymbol{F}$ denotes a solenoidal external force. The velocity $\boldsymbol{U}$ and the magnetic field $\boldsymbol{B}$ are expressed in units of a characteristic speed $U_{0}, \epsilon$ measures the relative strength of the Hall effect, and $\eta$ and $\nu$ are the (dimensionless) magnetic diffusivity and kinematic viscosity, respectively. Note that the measure of Hall effect $\epsilon$ can be written as

$$
\epsilon=\frac{L_{\text {Hall }}}{L_{0}}
$$

where $L_{0}$ is a characteristic length scale (the size of the box in our simulations is equal to $2 \pi$ ), and the Hall length

$$
L_{H a l l}=\frac{c}{\omega_{p i}} \frac{U_{A}}{U_{0}},
$$

is given in terms of the characteristic speed $U_{0}$ and the characteristic Alfvénic speed $U_{A}$. In particular, we are free to choose $U_{0}=U_{A}$ as our characteristic velocity, reducing $L_{\text {Hall }}$ to the ion skin depth.

Equation (5) is valid in a fully ionized plasma. We will work under this assumption without any loss of generality. In the more general case of partially ionized plasmas, the values of $L_{\text {Hall }}$ and $\epsilon$ are those reported by Sano \& Stone (2002), or in Mininni, Gómez, $\&$ Mahajan (2003b). Typical values of $\epsilon$ in astrophysics are also mentioned in these papers.

To keep in mind astrophysical scenarios, we just recall three examples. In a protostellar disk the Hall scale is larger than the dissipation scale typically by two orders of magnitude, but smaller than the largest scales of the system (Balbus \& Terquem 2001). Therefore we expect $\epsilon<1$ but with $L_{\text {Hall }}$ larger than the dissipation scale. In some dwarf nova disks and protoplanetary disks $\epsilon \approx 1$ (Sano \& Stone 2002). As previously mentioned, in neutron stars $\epsilon>1$ (Muslimov 1994).

The Hall-MHD system has three well-known ideal $(\eta=\nu=0)$ quadratic invariants

$$
\begin{aligned}
E & =\frac{1}{2} \int\left(U^{2}+B^{2}\right) d V, \\
H_{m} & =\frac{1}{2} \int \boldsymbol{A} \cdot \boldsymbol{B} d V, \\
K & =\frac{1}{2} \int(\boldsymbol{B}+\epsilon \boldsymbol{\omega}) \cdot(\boldsymbol{A}+\epsilon \boldsymbol{U}) d V .
\end{aligned}
$$

Here $E$ is the energy, $H_{m}$ is the magnetic helicity, and $K$ is the hybrid helicity, which replaces the cross helicity from magnetohydrodynamics. The vector potential $\boldsymbol{A}$ is defined by $\boldsymbol{B}=\nabla \times \boldsymbol{A}$, and $\boldsymbol{\omega}=\nabla \times \boldsymbol{U}$ is the vorticity. Conservation of these ideal invariants during the evolution of the system provides a check on the simulation.

\section{THE CODE}

The pseudospectral code used in Mininni, Gómez, \& Mahajan (2003b) was modified to run in a Beowulf cluster using MPI. We integrated the Hall-MHD equations (1)-(3) in a cubic box with periodic boundary conditions. The equations were evolved in time using a second order Runge-Kutta method. The total pressure $P_{T}=P+B^{2} / 2$ was computed in a self-consistent fashion at each time step to ensure the incompressibility condition $\nabla \cdot \boldsymbol{U}=0$ (Canuto, Hussaini, Quarteroni, $\&$ Zang 1998). In Fourier space, taking the divergence of equation (2) we obtain

$$
\widehat{P_{T}}(\boldsymbol{k})=\frac{i}{k^{2}} \boldsymbol{k} \cdot\left[(\widehat{\boldsymbol{U} \cdot \widehat{\nabla \boldsymbol{U}}})_{\boldsymbol{k}}-(\widehat{\boldsymbol{B} \cdot \widehat{\nabla \boldsymbol{B}}})_{\boldsymbol{k}}\right],
$$

where the hat denotes a spatial Fourier transform, and $\boldsymbol{k}$ is the wavenumber vector.

To satisfy the divergence-free condition for the magnetic field, the induction equation (1) was replaced by an equation for the vector potential

$$
\frac{\partial \boldsymbol{A}}{\partial t}=(\boldsymbol{U}-\epsilon \nabla \times \boldsymbol{B}) \times \boldsymbol{B}+\epsilon \nabla p_{e}+\eta \nabla^{2} \boldsymbol{A},
$$

where $p_{e}$ (electron pressure) was computed at each time step to satisfy the Coulomb gauge $\nabla \cdot \boldsymbol{A}=0$, solving an equation similar to equation (9).

We present results from different runs with $\eta=$ $0.05, \eta=0.02$, and $\eta=0.011$. For the first value of $\eta$, simulations with $64^{3}$ and $128^{3}$ spatial grid points were performed to check convergence. The rest of the simulations were made with $128^{3}$ grid points $(\eta=0.02)$, and $256^{3}$ grid points $(\eta=0.011)$. All the runs were made with magnetic Prandtl number $\nu / \eta=1$. Therefore, hereafter we will only consider a single Reynolds number (i.e. both kinetic and magnetic), defined as

$$
R=\frac{U L_{0}}{\eta},
$$

In the study of turbulent flows, the number

$$
R_{\lambda}=\frac{U \lambda}{\eta}
$$

constructed from Taylor's length scale $\left[\lambda=\left(\left\langle U^{2}\right\rangle /\left\langle\omega^{2}\right\rangle\right)^{1 / 2}\right]$ is often considered. Note that this definition of 
Taylor's micro-scale might differ from other definitions (for instance, in connection with experiments on fluid turbulence) in factors of order unity (Pope 2000). These two Reynolds numbers were respectively $R \approx 100$ and $R_{\lambda} \approx 20$ for the first value of $\nu$ and $\eta, R \approx 300$ and $R_{\lambda} \approx 40$ for the second one, and $R \approx 560$ and $R_{\lambda} \approx 60$ in the last case. The energy injection rate was approximately the same for all these runs.

The simulation begins by subjecting the NavierStokes equation to a stationary helical force $\boldsymbol{F}$ (given by eigenfunctions of the curl operator) operating at a macroscopic scale $k_{\text {force }}=3$ (Mininni, Gómez, \& Mahajan 2003b) to reach a hydrodynamic turbulent steady state. The resulting statistically steady state is characterized by a positive kinetic helicity. The relative helicity in runs with $R=300$ is $2 H_{k} /\left(\left\langle U^{2}\right\rangle\left\langle\omega^{2}\right\rangle\right)^{1 / 2} \approx 0.4$, and this value decreases slightly for larger Reynolds numbers. The kinetic helicity is defined as

$$
H_{k}=\frac{1}{2} \int \boldsymbol{U} \cdot \boldsymbol{\omega} d V .
$$

Once the hydrodynamic stage of the simulation reaches a steady state, a non-helical but small magnetic seed was introduced. This initial magnetic seed was generated by a $\delta$-correlated vector potential centered at $k_{\text {seed }}=13$ for the $R=100$ runs and $k_{\text {seed }}=35$ for the $R=300$ and $R=560$ simulations. The run was continued with the same external helical force in the Navier-Stokes equation, to study the growth of magnetic energy due to dynamo action.

Another set of simulations was made under the same conditions but with $\nu=\eta=0.02$ and $k_{\text {force }}=$ 10 ( $128^{3}$ grid points), to study the changes in the growth of the large scale magnetic field in the presence of the Hall effect. In this case the Reynolds numbers are smaller ( $R \approx 220$ and $R_{\lambda} \approx 20$ ), and the turbulence is weaker, since there are not enough modes in Fourier space for a direct cascade to develop properly. On the other hand, there are more Fourier modes to study the inverse cascade and the growth of large scale fields. The results of these simulations are discussed in Section 6.

In all our simulations the Kolmogorov's kinetic and magnetic dissipation length scales were properly resolved in the computational domain, i.e. we made sure that the dissipation wavenumbers remain smaller than the maximum wavenumber allowed by the dealiasing step, namely $k_{\max }=128 / 3$.

\section{MHD DYNAMOS}

In this section we briefly present the results from MHD simulations. The results are in good agreement with previous simulations of dynamo action under periodic boundary conditions (Meneguzzi, Frisch, \& Pouquet 1981; Brandenburg 2001). These simulations are intended for comparison with the Hall-MHD runs, and therefore some specific results of the MHD simulations are discussed in more detail in the following sub-sections.

\subsection{Magnetic energy evolution}

In Figure 1, we show the magnetic and kinetic energy as a function of time in MHD runs $(\epsilon=0)$ for different Reynolds numbers. The turnover time for $R=300$ is $\tau=2 \pi /\left(k_{\text {force }}\left\langle U^{2}\right\rangle^{1 / 2}\right) \approx 0.3$.

Two phases can be clearly identified in the evolution of the magnetic energy. After a first stage with exponential growth (which can be considered as the kinematic dynamo stage), the magnetic energy saturates and reaches equipartition with the kinetic energy (see Figure 1). In the first stage, the magnetic energy is still weak and the velocity field is not strongly affected by the Lorentz force. Note that during this exponential growth of magnetic energy, the kinetic energy remains approximately constant.

This kinematic dynamo stage can be understood at least qualitatively considering the mean field induction equation (Krause \& Rädler 1980)

$$
\frac{\partial \overline{\boldsymbol{B}}}{\partial t}=\nabla \times(\overline{\boldsymbol{U}} \times \overline{\boldsymbol{B}}+\alpha \overline{\boldsymbol{B}})+\eta_{e f f} \nabla^{2} \overline{\boldsymbol{B}} .
$$

Here the overline denotes mean field quantities, and

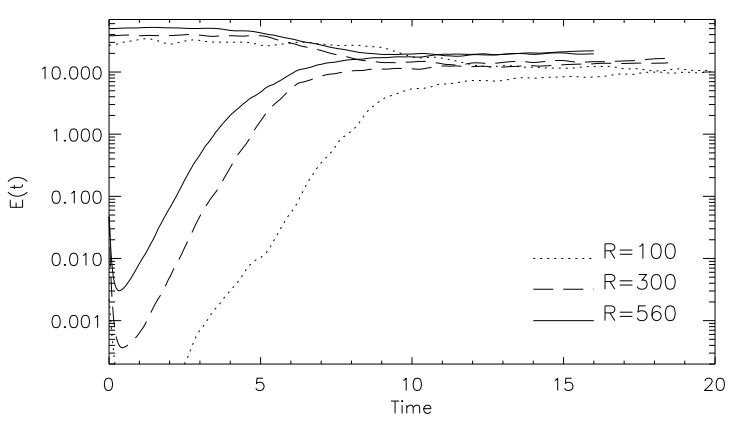

Fig. 1.- Kinetic energy $E_{k}$ (above), and magnetic energy $E_{m}$ (below) as a function of time $(\epsilon=0)$. 
$\eta_{\text {eff }}$ is the magnetic plus turbulent diffusivity. The MHD $\alpha$-effect (Pouquet, Frisch, \& Leorat 1976)

$$
\alpha=\frac{\tau}{3}(-\overline{\boldsymbol{u} \cdot \nabla \times \boldsymbol{u}}+\overline{\boldsymbol{b} \cdot \nabla \times \boldsymbol{b}}),
$$

represents the back-reaction of the turbulent motions in the mean field, and gives exponential growth of magnetic energy in the kinematic regime for helical turbulence. Here $\boldsymbol{u}$ and $\boldsymbol{b}$ are respectively the fluctuating velocity and magnetic fields, and $\tau$ is a typical correlation time for the turbulent motions. Attempts to measure this quantity in direct simulations were made by Cattaneo \& Hughes (1996), and Brandenburg (2001).

As the Reynolds number increases, the saturation field strength increases, although it seems to reach an asymptotic value. After the saturation, the magnetic energy keeps growing slowly on a resistive timescale (Brandenburg 2001). This late growth takes place mainly at large scales, as will be shown in the energy spectrum. As far as we know, there are no simulations of MHD dynamos with periodic boundary conditions showing generation of large scale fields on shorter times.

\subsection{Energy spectrum}

Figure 2 shows the kinetic and magnetic spectra at different times for a run with $R=300$. In the early stages the magnetic energy grows uniformly at all wave numbers. After the saturation $(t \approx 5)$ the emergence of a large-scale field can be clearly seen in the spectrum. At $t \approx 18.4$, when the system has already reached equipartition (see Figure 1), the magnetic energy at large scales (small wave numbers) still keeps growing, albeit slowly. As a result, the large scale magnetic field reaches super-equipartition with the kinetic energy. An excess of magnetic energy can be also observed at small scales.

The slope of the total (magnetic and kinetic) energy spectrum in the inertial range is consistent with Kolmogorov's $k^{-5 / 3}$ law and in good agreement with simulations of helical MHD turbulence with higher spatial resolutions. Kida, Yanase, \& Mizushima (1991) found that the total energy spectrum in MHD dynamo simulations is of the form

$$
E(k)=C_{K} \varepsilon^{2 / 3} k^{-5 / 3},
$$

where $\varepsilon$ is the total dissipation rate, and $C_{K}$ is a Kolmogorov's constant. Simulations at higher spatial resolutions (Haugen, Brandenburg \& Dobler 2003) seem to confirm this result although in some cases the spectrum tends to be a little shallower.

\subsection{Magnetic helicity}

The magnetic helicity $H_{m}$ is displayed in Figure 3. The initial magnetic field is non-helical, but during the dynamo process net magnetic helicity is generated with a sign opposite to that of the kinetic helicity. This helicity is located mostly in large scale structures, as will be shown in Section 6 .

From mean field equations we obtain for the large scale magnetic helicity (Mininni, Gómez, \& Mahajan 2003b)

$$
\frac{d \overline{H_{m}}}{d t}=2 \int\left(\alpha \bar{B}^{2}-\eta_{e f f} \overline{\boldsymbol{J}} \cdot \overline{\boldsymbol{B}}\right) d V
$$

where $\boldsymbol{J}=\nabla \times \boldsymbol{B}$ is the electric current density. This equation represents a transfer of magnetic helicity from small scales to large scales. The mean field helicity grows with the same sign as the $\alpha$ coefficient (opposite sign as the kinetic helicity). Our results are in good agreement with this relation, as well as previous simulations of MHD dynamo action (Brandenburg 2001; Mininni, Gómez, \& Mahajan 2003b).

Although this generation of magnetic helicity by dynamo action is expected to decrease as the magnetic Reynolds number $R_{m}$ increases, in simulations with higher Reynolds numbers the growth of magnetic helicity seems to reach an asymptotic value.

\section{HALL DYNAMOS}

To quantitatively assess the role of the Hall effect on dynamo action, we display results from runs with different values of $\epsilon$ : the MHD run $(\epsilon=0)$ and HallMHD runs with $\epsilon=0.066,0.1$ and 0.2 . We will focus on the Hall-enhanced dynamo regime (Mininni, Gómez, \& Mahajan 2003b). Note that all these values of $\epsilon$ correspond to dynamos where the Hall effect is only relevant in a fraction of the scales involved. The Hall inverse length scale for these runs is measured by $k_{\text {Hall }}=15,10$ and 5 respectively $\left(k_{\text {Hall }}=1 / \epsilon\right)$. All length scales smaller than the Hall scale are expected to be strongly affected by the Hall effect. The Kolmogorov's kinetic dissipation scale $\left[k_{\nu}=\left(\left\langle\omega^{2}\right\rangle / \nu^{2}\right)^{1 / 4}\right]$ is $k_{\nu} \approx 20$ when $R=100$, $k_{\nu} \approx 40$ when $R=300$, and $k_{\nu} \approx 75$ when $R=560$. 


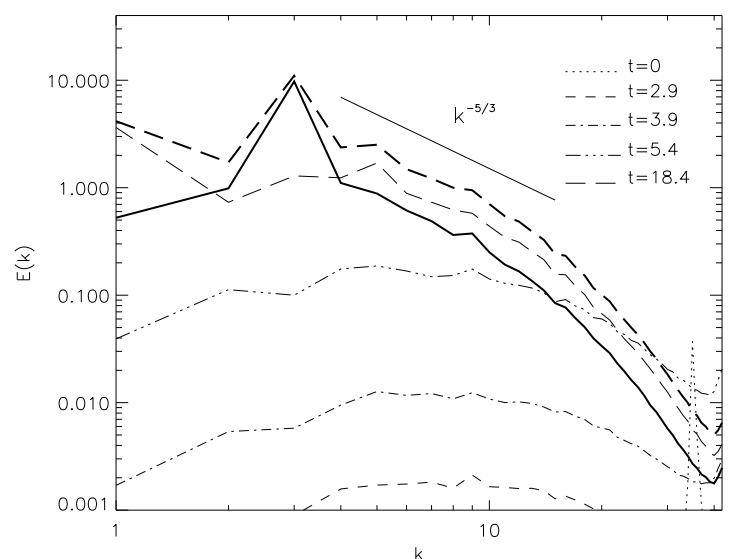

Fig. 2.- Mean kinetic energy spectrum (thick line), total energy spectrum (thick dashed line), and magnetic energy spectrum at different times $(\epsilon=0$ and $R=300$ ). The Kolmogorov's slope is shown as a reference.

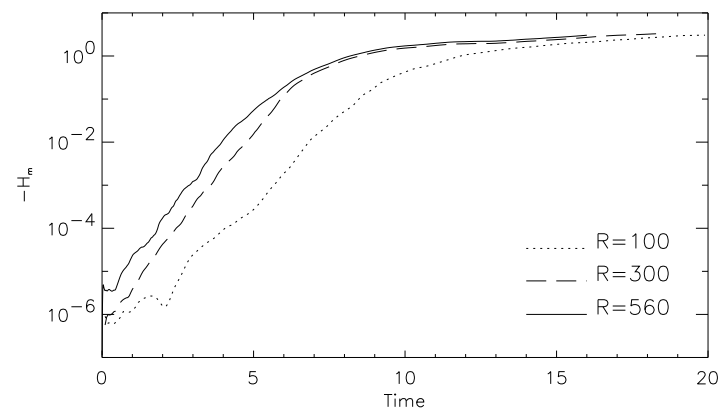

Fig. 3.- Magnetic helicity $\left(-H_{m}\right)$.

\subsection{Magnetic energy evolution}

Figure 4 shows the kinetic and magnetic energy as a function of time for the MHD and a Hall-MHD run with $R=300$ and $\epsilon=0.1$. At early times, the evolution of magnetic energy in MHD and Hall-MHD is similar. Using mean field theory, the induction equation for the mean magnetic field reduces to

$\frac{\partial \overline{\boldsymbol{B}}}{\partial t}=\nabla \times[(\overline{\boldsymbol{U}}-\epsilon \nabla \times \overline{\boldsymbol{B}}) \times \overline{\boldsymbol{B}}+\alpha \overline{\boldsymbol{B}}]+\eta_{e f f} \nabla^{2} \overline{\boldsymbol{B}}$,

with the Hall-MHD $\alpha$-effect now given by (Mininni, Gómez, \& Mahajan 2002)

$$
\begin{aligned}
\alpha= & \frac{\tau}{3}\left(-\overline{\boldsymbol{u}^{e} \cdot \nabla \times \boldsymbol{u}^{e}}+\overline{\boldsymbol{b} \cdot \nabla \times \boldsymbol{b}}-\right. \\
& \left.-\epsilon \overline{\boldsymbol{b} \cdot \nabla \times \nabla \times \boldsymbol{u}^{e}}\right) .
\end{aligned}
$$

Here $\boldsymbol{u}^{e}=\boldsymbol{u}-\epsilon \nabla \times \boldsymbol{b}$ is the fluctuating electron flow velocity. When the fluctuating magnetic field is weak, this expression reduces to equation (15) and the Hall effect can be dropped. Therefore, the first stage corresponds to a kinematic dynamo during which the Hall effect is negligible.

After this stage, and when the dynamo-generated magnetic fields are strong enough for the Hall effect to become non-negligible, the evolution changes and the magnetic energy keeps growing but at a different pace (see also Figure 5). A third stage can be identified, when the velocity field is affected by the Lorentz force and the dynamo reaches saturation. The kinetic energy drop is not as intense as in the MHD case, and the increase of magnetic energy in this final stage is larger than in the MHD case for moderate values of $\epsilon$. Finally, a state with more magnetic energy than its MHD

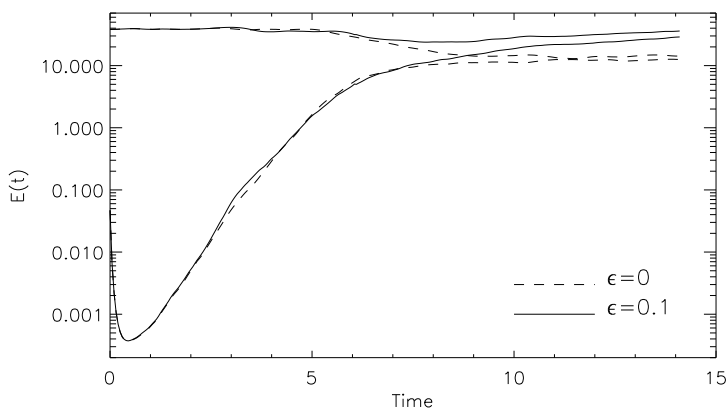

Fig. 4.- Magnetic (below) and kinetic energy (above) as a function of time for two runs with $\epsilon=0.1$ and $\epsilon=0(R=300)$. 
counterpart is reached (by a factor 2.3 when $R=300$ and $\epsilon=0.1$ ).

Figure 5 shows the evolution for a Hall-MHD run with $R=300$ and $\epsilon=0.2$. Here, after the exponentially growing stage, the magnetic field saturates at an amplitude smaller than the previous run. Note that equipartition between the kinetic and magnetic energy is not reached.

When a turbulent stationary state is attained, the sum of magnetic and kinetic energy is not equal to the initial kinetic energy. This is related to the fact that, when the magnetic seed is introduced, a new channel for energy dissipation arises,

$$
\frac{d E}{d t}=-\nu \int \omega^{2} d V-\eta \int J^{2} d V,
$$

As in previous simulations (Mininni, Gómez, \& Mahajan 2003b), it is found that the final energy reached for the Hall-MHD runs is larger than the value obtained for the MHD runs, revealing that Hall-MHD dynamos can be more efficient (in the sense that they generate more magnetic energy and dissipate less total energy).

Figure 6 shows the maximum value attained by the magnetic energy as a function of $\epsilon$ for several simulations with different Reynolds numbers and scale separations. When $R=560$ the final amplitude reached by the magnetic energy is unknown. Given the stringent quadratic Courant-Friedrich-Lewy (CFL) condition imposed by dispersive waves in Hall-MHD, simulations were only carried up to saturation of the dynamo. The maximum value of the energy in Figure 6 is normalized with the value obtained in an MHD run with the same Reynolds numbers and initial kinetic en-

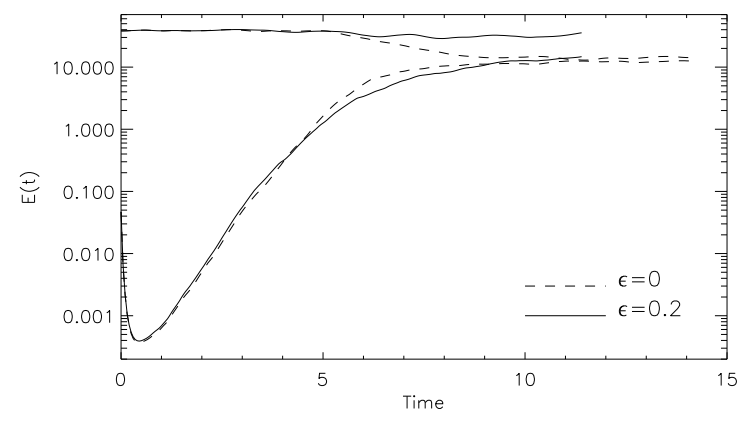

Fig. 5.- Magnetic (below) and kinetic energy (above) as a function of time for two runs with $\epsilon=0.2$ and 0 $(R=300)$. ergy and helicity. As previously mentioned, considering the three different regimes of the Hall dynamo discussed in Mininni, Gómez, \& Mahajan (2003b), we focus on the Hall-enhanced case. As the Reynolds numbers are increased, the efficiency of the Hall-MHD dynamo grows. Also, the value of $\epsilon$ at which maximum efficiency is obtained, decreases as the Reynolds numbers are increased. Note that the growth of the efficiency of the Hall-MHD dynamo with increasing scale separation was predicted analytically by Mininni, Gómez, \& Mahajan (2002). The shift of most efficient $\epsilon$ to smaller values as $R$ increases is also obtained from analytical estimates (Mininni, Gómez, \& Mahajan 2004).

\subsection{Energy spectrum}

Figure 7 shows the kinetic, magnetic, and total energy spectra at different times for $R=300$ and $\epsilon=0.1$. Barring $\epsilon$, all the parameters and initial conditions in this run are the same as those corresponding to Figure 2. Therefore, a direct comparison between the evolution of both spectra can be made. During the first few time steps, the evolution is similar to the MHD run, with the entire magnetic spectrum growing at almost the same rate. The difference observed in Mininni, Gómez, \& Mahajan (2003b), that the largescale magnetic field is slightly larger than in its MHD counterpart, is now increased as a result of the larger Reynolds number and larger scale separation.

The rate of increase of the large scale magnetic field changes in the presence of the Hall effect. While in MHD the build-up of this field proceeds on a resistive timescale, in Hall-MHD it grows faster. Note that the magnetic energy in the shell $k=1$ in Hall-MHD $(\epsilon=0.1)$ simulation at $t=14.1$ is a factor of 2 larger than the magnetic energy in the same shell in the MHD run at $t=18.4$. Also, the kinetic energy in the same shell is larger. This can be also observed in Figure 8, which shows the energy contained in the large scale magnetic field (in the shell $k=1$ ) as a function of time for several values of $\epsilon$. However, the filling factor $\left(\left\langle\bar{B}^{2}\right\rangle /\left\langle B^{2}\right\rangle\right)^{1 / 2}$ is smaller as $\epsilon$ is increased. At $t=14$ the filling factor is $\approx 0.23$ for $\epsilon=0, \approx 0.17$ for $\epsilon=0.1$, and $\approx 0.1$ for $\epsilon=0.2$,

Note also that, while the MHD spectrum shows super-equipartition at small scales (the magnetic energy is larger than the kinetic energy at large wave numbers), the Hall-MHD leads to equipartition at these scales. Both the evolution of the large scale 


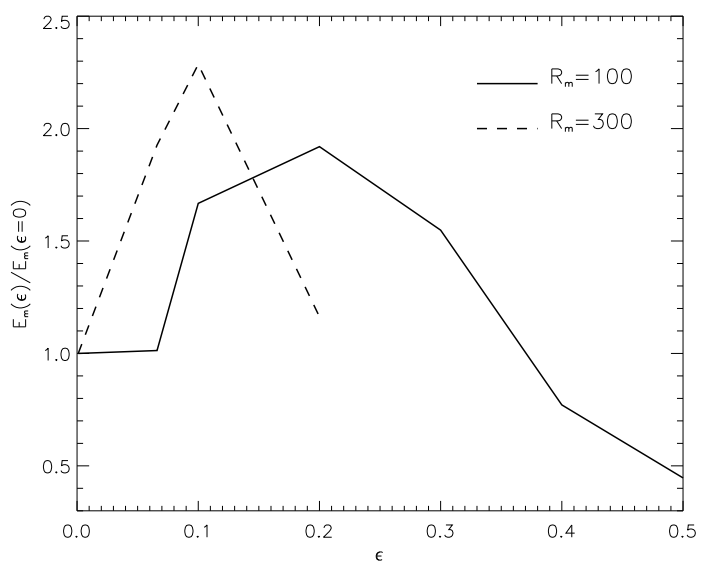

Fig. 6. $-E_{m} / E_{m}(\epsilon=0)$ as a function of $\epsilon$ for runs with $R=100$ and $R=300$. The values of $E_{m}$ correspond to the magnetic energy at the saturation level, while $E_{m}(\epsilon=0)$ is the magnetic energy for an MHD run

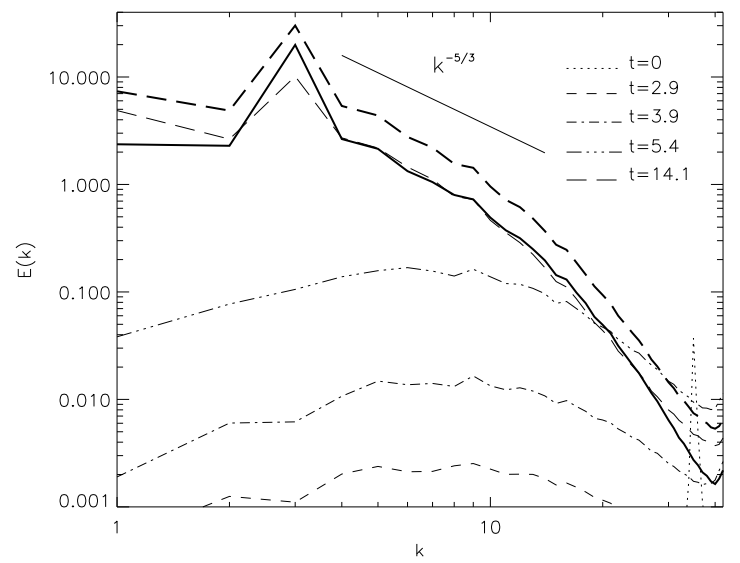

Fig. 7.- Mean kinetic energy spectrum (thick line), total energy spectrum (thick dashed line), and magnetic energy spectrum at different times $(\epsilon=0.1$ and $R=300)$. and small scale magnetic fields are therefore clearly affected by the Hall effect, even though the Hall effect operates effectively only at small scales.

Figure 9 shows the spectrum for $R=300$ and $\epsilon=$ 0.2 . In this case all wave numbers larger than $k_{\text {Hall }}=$ 5 are affected by the Hall effect. However, the total energy spectrum in the saturated state seems to obey a Kolmogorov type law, although the Hall length scale is placed in the middle of the inertial range.

Figure 10 shows the compensated energy spectrum $E(k) /\left(\varepsilon^{2 / 3} k^{-5 / 3}\right)$ for higher spatial resolution runs with $R=560$ and $\epsilon=0$ and 0.1 , using $256^{3}$ grid points. If the spectrum obeys a Kolmogorov type law, the compensated spectrum should be flat over a certain range, and the amplitude of the spectrum in this range gives the Kolmogorov constant $C_{K}$. The mild hump observed for the total energy before entering the dissipative range, might be indicative of the presence of a "bottleneck effect" for the energy cascade, as was recently discussed by Haugen, Brandenburg \& Dobler (2003).

The spectra displayed in Figure 10 correspond to the era when the dynamo is saturated. The first example (Fig. 10(a)) represents the MHD limit (i.e. $\epsilon=0$ ), while the second one (Fig. 10(b)) has $k_{\text {Hall }}=10$ $(\epsilon=0.1)$. All the length scales smaller than $1 / k_{\text {Hall }}$ are expected to be dominated by the Hall effect. For the MHD spectrum, the Kolmogorov constant $C_{K} \approx$ 1.39 , a value slightly larger than the one found by Haugen, Brandenburg \& Dobler (2003), $C_{K} \approx 1.3$ (see also Haugen, Brandenburg \& Dobler (2004)), but smaller than the one obtained by Kida, Yanase, \& Mizushima (1991) $\left(C_{K} \approx 2.1\right)$. No clear change in the slope can be identified in the Hall-MHD case,

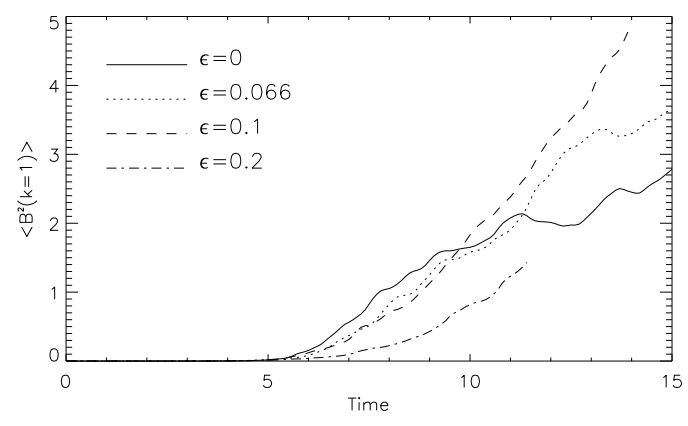

Fig. 8.- Magnetic energy in the shell $k=1$ in Fourier space as a function of $\epsilon$ and time. 
and the spectrum is compatible with a Kolmogorov type law in its inertial range. However, the spectrum could really be a little shallower. Higher spatial resolution simulations are needed to settle this point. In this case the Kolmogorov's constant turns out to be $C_{K} \approx 1.66$, somewhat larger than its MHD counterpart. The Kolmogorov's dissipation wave numbers in the last stages of these runs are $k_{\nu} \approx k_{\eta} \approx 70$ [where $\left.k_{\eta}=\left(\left\langle J^{2}\right\rangle / \eta^{2}\right)^{1 / 4}\right]$.

\subsection{Magnetic helicity}

In Mininni, Gómez, \& Mahajan (2003b), the Hall effect was observed to inhibit the creation of net magnetic helicity by the dynamo process. This effect is enhanced as we increase the Reynolds numbers. While the MHD dynamo is an efficient generator of magnetic helicity with most of this helicity concentrated in the larger scales, the Hall dynamo is somewhat sluggish; the growth of net magnetic helicity is slower and in some cases oscillates around zero (see Figure 11.) This result is in good agreement with theoretical estimates suggesting that in the presence of the Hall effect reconnection events are faster (Priest \& Forbes 1998; Ji 1999), and therefore dissipate less magnetic helicity (see Section 6).

\subsection{Kinetic helicity}

In MHD, relative kinetic helicity is known to change only slightly during dynamo action (Brandenburg 2001). In all these simulations, kinetic energy and kinetic helicity are injected at the same length scale by the stirring force acting at $k_{\text {force }}=3$. For homogeneous hydrodynamic turbulence, kinetic helicity directly cascades to smaller scales. On dimensional grounds (Moffat 1978), the spectrum of kinetic helicity in the inertial range is also expected to follow Kolmogorov's law (Cheng et al. 2003; Gómez \& Mininni 2004)

$$
H_{k}(k)=C_{H} k_{h e l} \varepsilon^{2 / 3} k^{-5 / 3},
$$

where $C_{H}$ is another Kolmogorov constant, and $k_{h e l}$ is the scale where kinetic helicity is injected. Using the inertial range kinetic energy expression $\left(C_{K}\right.$ is the standard Kolmogorov's constant )

$$
E_{k}(k)=C_{K} \varepsilon^{2 / 3} k^{-5 / 3},
$$

the ratio between the total kinetic helicity and kinetic energy in hydrodynamic turbulence come out to be

$$
\frac{H_{k}}{E_{k}}=\frac{\int \boldsymbol{U} \cdot \boldsymbol{\omega} d V}{\int U^{2} d V} \approx k_{h e l},
$$

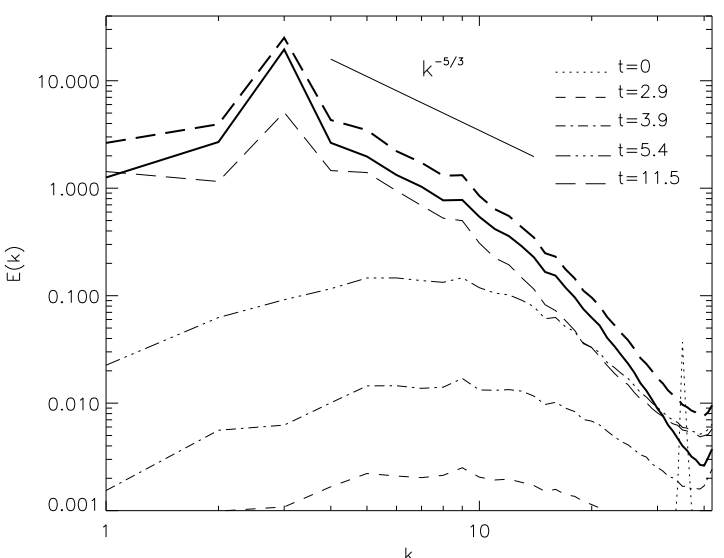

Fig. 9.- Mean kinetic energy spectrum (thick line), total energy spectrum (thick dashed line), and magnetic energy spectrum at different times $(\epsilon=0.2$ and $R=300)$.
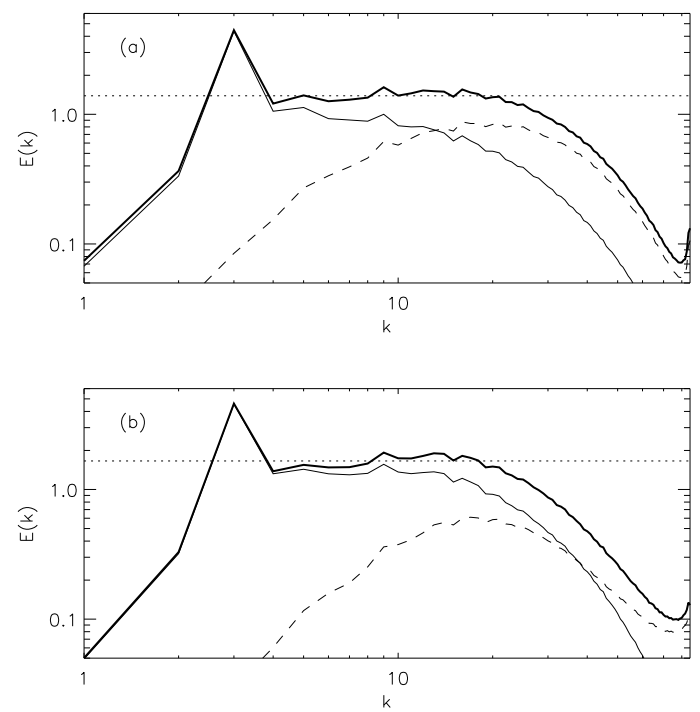

Fig. 10.- Total compensated energy spectrum $k^{5 / 3} \varepsilon^{-2 / 3} E_{k}$ (thick line), kinetic energy spectrum (thin line), and magnetic energy spectrum (dashed line) at $t=5.5$ for a run with (a) $\epsilon=0$, and (b) $\epsilon=0.1$ ( $R=560,256^{3}$ grid points). 
where $k_{\text {hel }}=k_{\text {force }}$ in our case. What happens after we introduce the magnetic seed?

Figure 12 shows the ratio $H_{k} / E_{k}$ for the MHD and the Hall-MHD runs with $R=300$. The evolution of the relative kinetic helicity is similar. In the MHD run $H_{k} / E_{k} \approx 2.5$, a value close to $k_{\text {force }}=3$. Note that, although the total kinetic energy (and the kinetic helicity) decreases during the time evolution as a result of the increasing Lorentz force, the ratio $H_{k} / E_{k}$ remains nearly constant. On the other hand, this ratio grows with $\epsilon$ in the Hall-MHD runs. The growth takes place just after the exponentially growing stage, when a large scale magnetic field is developing in the box. This result suggests that a new source of kinetic helicity has appeared, and is in good agreement with theoretical estimates suggesting that the Hall effect introduces handedness in the fluid motions (Mininni, Gómez, \& Mahajan 2004). However, we want to point out that this handedness does not, by itself, generate a net $\alpha$-effect.

As previously mentioned, the relative kinetic helicity $H_{k} /\left(\left\langle U^{2}\right\rangle\left\langle\omega^{2}\right\rangle\right)$ in Hall-MHD also shows the same behavior, changing from a relative kinetic helicity of 0.4 when $\epsilon=0$ up to about 0.6 when $\epsilon=0.1$.

\section{LARGE SCALE MAGNETIC FIELD GEN- ERATION}

In this section we present MHD and Hall-MHD results for an external force located at $k_{\text {force }}=10$. Simulations were carried with $\nu=\eta=0.02$ and $128^{3}$ grid points. In this case the Reynolds numbers are smaller ( $R \approx 220$ and $R_{\lambda} \approx 20$ ), and the turbulence is weaker, since there are not enough modes in Fourier space for a direct cascade to develop properly. On the other hand, there is more room for the generation of a large scale

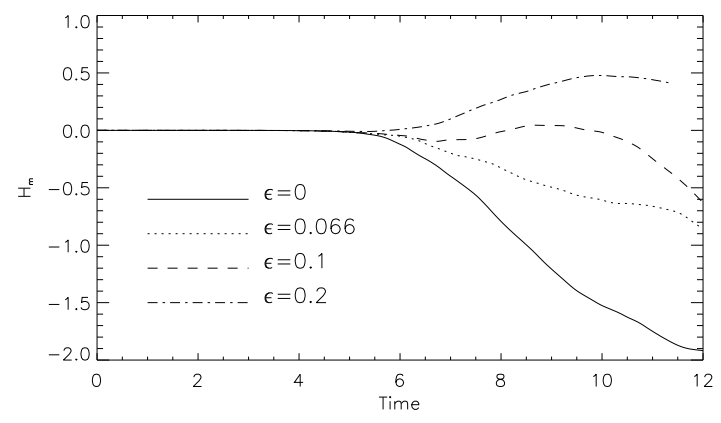

Fig. 11.- Magnetic helicity for $\epsilon=0,0.066,0.1$, and $0.2(R=300)$. magnetic field through inverse cascade. Simulations were performed for $\epsilon=0,0.1$, and 0.2 , corresponding respectively to the MHD case, $k_{\text {Hall }}=10$ and $k_{\text {Hall }}=5$.

Figure 13 shows the evolution of the magnetic and kinetic energy in the MHD simulation. After saturation, a large scale magnetic field grows on a resistive time scale, as will be shown in the energy spectrum and was previously observed in large scale dynamo simulations (Brandenburg 2001). Note that the system finally reaches a state of super-equipartition, i.e. a level of magnetic energy which is larger than the kinetic energy. Figure 14 shows its counterpart when $\epsilon=0.1$ and 0.2 . In the simulation with $\epsilon=0.1$, the growth of magnetic energy after the saturation is clearly faster, and the system reaches a final state with more magnetic energy than in the MHD run.

Figures 15 and 16 show the energy spectrum at different times for runs with $\epsilon=0$ and $\epsilon=0.1$, respectively. Note that in both simulations, the magnetic energy at intermediate scales $(2<k<8)$ starts to decay after saturation $(t>3.5)$, while magnetic energy at the largest scale $(k=1)$ keeps growing. As a result, after $t=8$ the system reaches the state of superequipartition in the MHD case. This is even more clear in Fourier space (Figure 15), where magnetic energy in the shell $k=1$ is two orders of magnitude larger than kinetic energy at $t=17.5$. An excess of magnetic energy can also be observed at small scales.

In the Hall-MHD case, we observe again a faster growth of the large scale magnetic field (Figure 16), but with a final state with super-equipartition only at large scales. Moreover, the kinetic energy in the shell $k=1$ is one order of magnitude larger than in the MHD case, and at small scales we obtain sub-

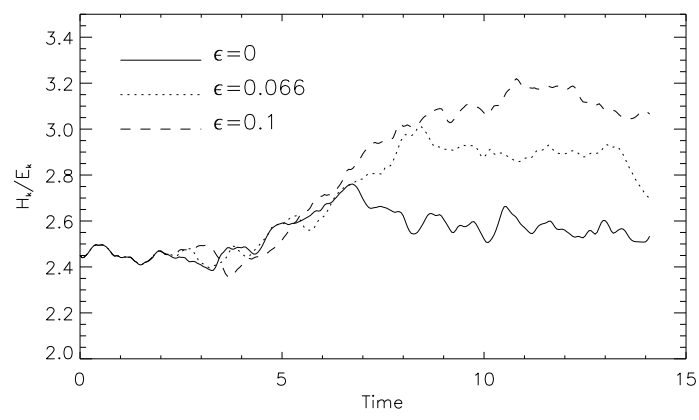

Fig. 12.- $H_{k} / E_{k}$ for $\epsilon=0,0.066$, and 0.1 . 


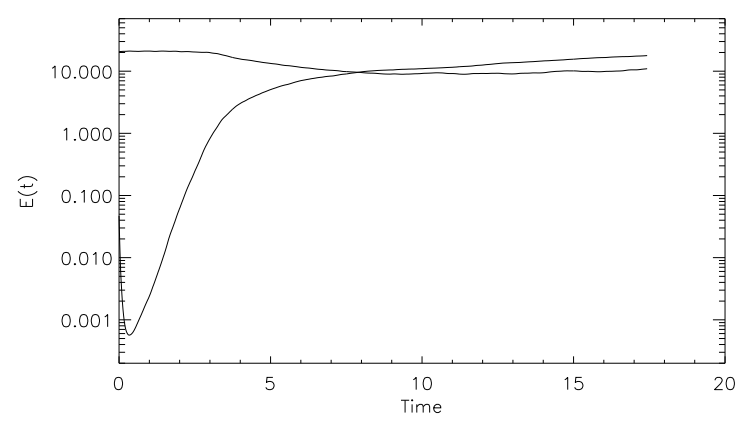

Fig. 13.- Magnetic (below) and kinetic energy (above) as a function of time for $\epsilon=0$ and $k_{\text {force }}=$ 10 .

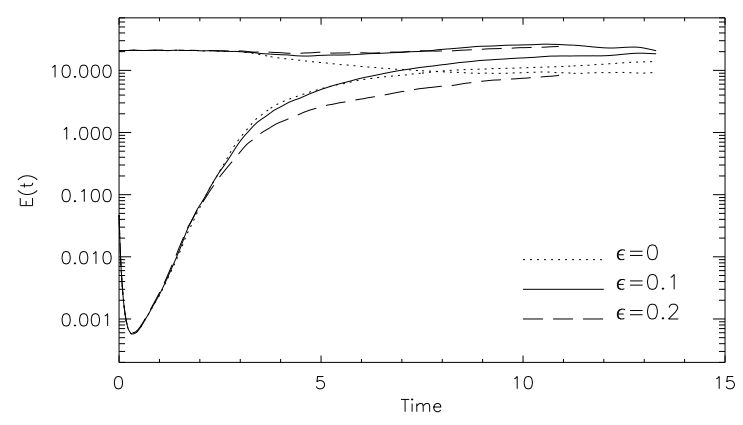

Fig. 14.- Magnetic (below) and kinetic energy (above) as a function of time for two runs with $\epsilon=0.2$, 0.1 and $0\left(k_{\text {force }}=10\right)$. equipartition between magnetic and kinetic energy.

Figure 17 shows the magnetic helicity spectrum as a function of time for different values of $\epsilon$. As mentioned in Section 4, the $\alpha$ effect creates magnetic helicity of a sign opposite to that of kinetic helicity at large scales. This effect is balanced by the creation of an opposite amount of magnetic helicity at small scales. Therefore, the diffusion preferentially destroys the short-scale magnetic helicity in reconnection events, leaving a net helicity of opposite sign at large scales (Brandenburg 2001). Mininni, Gómez, \& Mahajan (2003b) suggested that while the HallMHD dynamo process also creates equal and opposite amounts of magnetic helicity at large and at small scales, the dissipation of magnetic helicity at small scales is less efficient as $\epsilon$ is increased. Figure 17 shows that when the Hall effect is present, even at late times an excess of positive magnetic helicity at small scales $(k \geq 10)$ can be readily identified in the spectra.

\section{DISCUSSION}

In this paper we have presented the results of direct numerical simulations of turbulent dynamo action in Hall-MHD. We find that with increasing Reynolds number and scale separation, the Hall MHD dynamo works more efficiently when the Hall length is close but larger than the dissipation scale (Hall-enhanced regime). For larger values of $\epsilon$ the Hall MHD dynamo is less efficient. In addition, the value of $\epsilon$ (which measures the strength of the Hall term) at which the dynamo is most efficient decreases at higher Reynolds numbers.

An acceleration of the process responsible for the growth of a large scale magnetic field is observed at moderate values of $\epsilon$. Although these simulations are made at Reynolds numbers which are far away from realistic values for astrophysical plasmas, the results obtained are encouraging; the dynamos tend to work better at high Reynolds numbers

By calculating the magnitude and nature of the generated magnetic field as the amplitude of the Hall term is varied, we obtain new evidence showing that the Hall dynamo can be fundamentally different from its classical MHD counterpart.

Use of the Beowulf cluster Bocha at Departamento de Física, FCEN, UBA is acknowledged. The authors gratefully acknowledge A. Brandenburg and A. Pouquet for very fruitful and enlightening comments while 


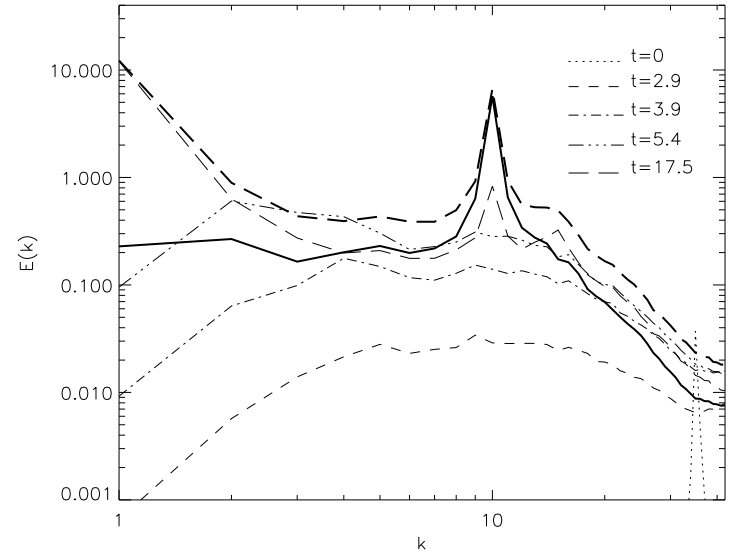

Fig. 15.- Mean kinetic energy spectrum (thick line), total energy spectrum (thick dashed line), and magnetic energy spectrum at different times $(\epsilon=0$ and $\left.k_{\text {force }}=10\right)$.

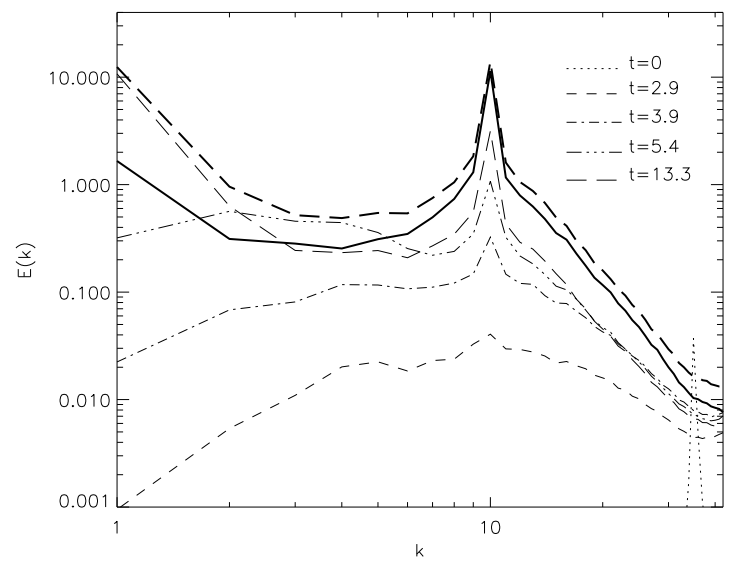

Fig. 16.- Mean kinetic energy spectrum (thick line), total energy spectrum (thick dashed line), and magnetic energy spectrum at different times $(\epsilon=0.1$ and $k_{\text {force }}=10$ ). reviewing the manuscript, and to the Abdus Salam International Centre for Theoretical Physics, were the initial stages of this study were performed. Research of SMM was supported by US DOE contract DEFG03-96ER-54366. Research of DOG and PDM has been partially funded by grant X209/01 from the University of Buenos Aires and by grant PICT 03-9483 from ANPCyT. PDM is a fellow of CONICET, and DOG is a member of the Carrera del Investigador Cientifico of CONICET.

\section{REFERENCES}

Balbus, S.A. \& Terquem, C. 2001, ApJ, 552, 235

Blackman, E.G. \& Field, G.B. 1999, ApJ, 521, 597

Braginskii, S.I. 1965, Rev. Plasma Phys., 1, 205

Brandenburg, A., \& Subramanian, K. 2000, Astron. \& Astrophys., 361, L33

Brandenburg, A. 2001, ApJ, 550, 824

Canuto, C., Hussaini, M.Y., Quarteroni, A., \& Zang, T.A. 1988, Spectral Methods in Fluid Dynamics (Berlin: Springer-Verlag)

Galanti, B., Kleeorin, N., \& Rogachevskii, I. 1994, Phys. of Plasmas, 1, 3843

Cattaneo, F. \& Hughes, D.W. 1996, Phys. Rev. E, 54, R4532

Chen, Q., Chen, S., \& Eyink, G. 2003, Phys. Fluids, 15,361

Gómez, D. \& Mininni, P. 2004, Phys. A, in press.

Haugen, N.E.L., Brandenburg, A., \& Dobler, W. 2003, ApJ, 597, L141

Haugen, N.E.L., Brandenburg, A., \& Dobler, W. 2004, Phys. Rev. E, 70, 016308

Heintzmann, H. 1983, JETP, 57, 251

Helmis, G. 1968, Mber. Dtsch. Akad. Wiss. Berlin, 10, 280

Helmis, G. 1971, Beitr. Plasma Physik, 11417

Ji, H. 1999, Phys. Rev. Lett., 83, 3198

Kida, S., Yanase, S., \& Mizushima J. 1991, Phys. Fluids, A3, 457 
Krause, F. \& Rädler, K.-H., 1980, Mean-Field Magnetohydrodynamics and Dynamo Theory, (GDR: Pergamon Press)

Meneguzzi, M., Frisch, U., \& Pouquet, A. 1981, Phys. Rev. Lett., 47, 1060

Mininni, P.D., Gómez, D.O., \& Mahajan, S.M. 2002, ApJ, 567, L81

Mininni, P.D., Gómez, D.O., \& Mahajan, S.M. 2003a, ApJ, 584, 1120

Mininni, P.D., Gómez, D.O., \& Mahajan, S.M. 2003b, ApJ, 587, 472

Mininni, P.D., Gómez, D.O., \& Mahajan, S.M. 2004 (in preparation).

Mirnov, V.V., Hegna, C.C., \& Prager, S.C. 2003, Plasma Phys. Rep., 29, 566

Moffat, H.K. 1978, Magnetic field generation in electrically conducting fluids (Cambridge: Cambridge University Press)

Muslimov, A.G. 1994, MNRAS, 267, 523

Pope, S.B. 2000, Turbulent flows (Cambridge: Cambridge University Press)

Pouquet, A., Frisch, U., \& Leorat, J. 1976, J. Fluid Mech., 77, 321

Priest, E. \& Forbes, T. 1998, Magnetic Reconnection (Cambridge: Cambridge Univ. Press)

Rheinhardt, M. \& Geppert, U. 2002, Phys. Rev. Lett., 88,101103

Sano, T. \& Stone, J.M. 2002, ApJ, 570, 314.

Zweibel, E.G. 1988, ApJ, 329, 384

This 2-column preprint was prepared with the AAS LATEX macros v5.0.
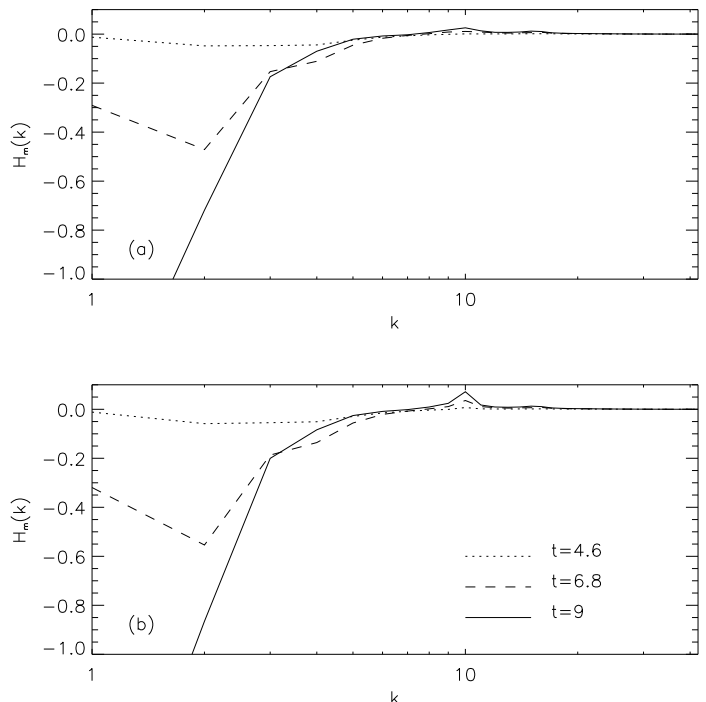

Fig. 17.- Magnetic helicity spectra for: (a) $\epsilon=0$, and (b) $\epsilon=0.2(R=300)$. 